\title{
MANAGING A PATIENT WAITING LIST WITH TIME-DEPENDENT PRIORITY AND ADVERSE EVENTS
}

\author{
DAIKI MIN ${ }^{1}$ AND YUEHWERN YIH ${ }^{2}$
}

\begin{abstract}
This paper addresses the problem of managing a waiting list for elective surgery to decide the number of patients selected from the waiting list and to schedule them in accordance with the operating room capacity in the next period. The waiting list prioritizes patients not only by their initial urgency level but also by their waiting time. Selecting elective surgery patients requires a balance between the waiting time for urgent patients and that for less urgent patients. The problem is formulated as an infinite horizon Markov Decision Process. Further, the study proposes a scheduling procedure based on structural properties of an optimal policy by taking a sampling-based finite horizon approximation approach. Finally, we examine the performance of the policy under various conditions.
\end{abstract}

Keywords. Waiting list management, time-dependent priority, adverse events while waiting, Markov Decision Process.

Mathematics Subject Classification. 90C40.

\section{INTRODUCTION}

This work is motivated mainly by previous empirical research suggesting that the waiting time for urgent patients is significantly shorter than that for nonurgent patients $[30,31,35]$. The delayed access to surgery causes the deterioration

Received November 8, 2012. Accepted October 15, 2013.

1 Assistant Professor, College of Business Administration, Ewha Womans University,

52 Ewhayeodae-gil, Seoul, 120-750, Korea. dmin@ewha.ac.kr

2 Professor, School of Industrial Engineering, Purdue University, 315 N. Grant Street, West

Lafayette, IN, 47906, USA. yih@purdue.edu 
of the patient's condition, which can increase the likelihood of adverse events such as emergency admission, death or a change of the attending physician. For example, in the case of elective cholecystectomy, the average weekly rate of emergency procedures is $2.7 \%$ for patients waiting 20 weeks or more, but it is $0.9 \%$ for those waiting less than 20 weeks [30]. In addition, previous studies have mentioned the risk of death or being removed from a surgical waiting list before surgery $[15,29]$. The removal of patients from the waiting list affects the waiting time for the others on the waiting list. This indicates a need for carefully considering the probability of adverse events together with the waiting time for patients when building a surgery schedule.

This paper refers to the patient pathway for elective coronary artery bypass grafting (CABG) surgery found in Sobolev and Kuramoto ([30]. pp. 17-18). Patients are placed on a waiting list if they cannot be immediately added to the current operating room schedule, and the patient remains on the list until his/her operating room time is scheduled. Every week, each surgical service group selects some patients from its waiting list on the basis of priority and places the selected patients on the operating room schedule for the next week. Such a scenario is analogous to the model proposed by Gerchak et al. [8], in which the scheduler has to determine how many requests for elective surgery to admit from the waiting list at the beginning of each day when the capacity is shared with emergency demands.

A problem associated with such a scenario is to decide how many of the patients on the waiting list should be assigned for the next service period (i.e., block time). Assigning too many patients to the block time is likely to lead to overtime hours. On the other hand, planning too few patients to cover the regular capacity may delay the access to patient care. In this regard, this paper aims to determine the appropriate number of patients selected for the next available capacity in consideration of an observed waiting list rather than booking a set of patients. In particular, we attempt to strike a balance between the waiting times for urgent patients and that for non-urgent patients by integrating a patient priority that is determined not only by the urgency level but also by the waiting time.

In Section 2, we first review the literature on patient waiting lists and related them to this work in the context of surgery scheduling problems. In Section 3, we formulate the problem as an infinite horizon MDP (Markov Decision Process). The need to record the dynamic status of patients explodes the problem size in terms of the state space and the corresponding solution space. It is well known that the state explosion limits the applicability of an MDP model in practice. To overcome this obstacle, we exploit structural properties to facilitate understanding of the characteristics of the value function and the optimal policy. Based on the theoretical establishments we then proceed to propose a solution procedure that relies on sampling-based finite-horizon approximation (Sect. 4). The algorithm estimates the optimal value and provides a non-stationary randomized policy in an online manner. Section 5 presents the results of the numerical experiments, which analyze how significantly the dynamic patient status affects the resulting solution in comparison with decisions that ignore the dynamics. 


\section{Literature REVIEW}

Building a waiting list according to patient priority or urgency level is widely perceived as an effective way to facilitate access to surgery [13]. Patient priority determines the relative position of each patient on the waiting list. Although there is no universal method for prioritizing patients, this study follows the concept proposed by Testi et al. [33], who prioritized patients by the priority score defined by $c \times \tau$, where $\tau$ indicates waiting time and $c$ refers to the initial urgency level for each patient based on his/her medical and social needs for treatment. Thus, the patient priority changes as the waiting time increases. Much research has indicated that the definition of patient priority should include the waiting time, because a long waiting time may lead to loss of opportunity for quality care. Readers can refer to MacCormick et al. [19] and Mullen [22] for a survey of prioritizing elective patients.

While some variants have been considered in the literature, most have approached the surgery scheduling problem by using the block capacity to assign patients until the anticipated total duration of all surgical procedures is not over the planned capacity $[6,11,20]$. The newsvendor model can effectively address the decision problems by considering the trade-off between the cost of idle capacity and the cost of overtime work $[18,24,32]$. Despite its effectiveness in terms of resource use, the newsvendor approach is limited in that it cannot address the patient waiting time.

Recent studies have included the waiting time in their models. Everett [7] proposed a simulation-based decision support system for a public hospital that evaluates the capacity feasibility for prioritized patients who are scheduled from a waiting list. Vasilakis et al. [35] evaluated the performance of pooling referrals comparing to the several individual waiting lists. In general, pooling referrals reduces the amount of time needed to schedule the surgery, but it can dramatically increase the amount of time the patient spends waiting for the surgery for nonurgent patients. Sobolev et al. [31] conducted a simulation study to compare two methods for booking elective surgery: booking from waiting lists and the direct booking of the surgery dates at the time of the decision. They evaluated cancellations and the patient waiting time and demonstrated that booking from a waiting list can reduce cancellations among urgent patients but that it can increase the waiting time for non-urgent patients.

Under the scheme of balancing delayed access to surgery with resource utilization, this work formulates the problem using a MDP model. We now provide a brief review of the literature considering patient priority or multiple types of patients in their MDP models. In addition to Gerchak et al. [8], who ignored patient priority in their model, most recent papers relevant to our work include Green et al. [9], Gupta [10], and Patrick et al. [25]. Green et al. [9] addressed a booking problem in which outpatients share a diagnostic resource with inpatients. Gupta [10] proposed a model for an elective surgery booking control problem with multiple patient priority, but the model left the multi-priority model for the future research area. 
A model given by Patrick et al. [25] attempted to book all patients with multiple types of urgency levels to fixed scheduling slots of a diagnostic resource within the maximum recommended waiting time.

Our work mainly differs from these studies in that problems associated with booking admissions involve the assignment of patients to appointment slots within a date that has fixed and same length. Such fixed time slots, which have typically been modeled as an appointment scheduling problem $[9,10,25]$, are not appropriate for the problem of scheduling surgery patients with random durations, because it is difficult to determine the number of patients who could receive their surgery during the block time.

Moreover, our work addresses the time-dependent patient priority, which little research has focused on. Although Patrick et al. [25] addressed the problem of scheduling multi-priority patients, their model's underlying scenario is different from that in this paper. First, the priority given to a patient at the beginning is not affected by his or her waiting time. Simulation studies proposed by Vasilakis et al. [35] and Sobolev et al. [31] demonstrated that the patient priority should change over time by showing that the time-evolving priority prevents the waiting time for non-urgent patients from increasing tremendously. Second, because they ignored waiting costs when patients wait less than the maximum recommended waiting time, low-priority patients face delayed access to their treatment until the recommended waiting time. In some cases, however, such delays can increase the likelihood of low-priority patients becoming emergency patients or even die before their waiting time reaches the maximum allowance ([30], pp. 117-140). Min and Yih [21] extended the work given by Gerchak et al. [8] to a multi-priority model, but their model also assumes a static patient priority. In line of the research interest, this study assumes that patient priority increases over time.

Further, this study contributes to the literature by considering the probability of adverse events while waiting. Only few papers have investigated the adverse events in their outpatient appointment scheduling problems in the forms of lateness of doctor arrivals [16], no-shows [23] or demand cancellations [17]. However, adverse events in outpatient appointment problems are very different from those in managing waiting lists for surgery. No-shows and cancellations in outpatient appointment problems are inherent in population behavior and are generally independent of outpatient appointment schedules. Some adverse events in the problem of managing waiting lists, however, may be prevented by shortening the waiting time.

\section{THE MODEL}

We consider a system that observes a list of patients waiting for surgery at the beginning of each week. Given such a waiting list, the surgical service group determines the number of patients scheduled for the next available operating room time. We formulate this problem as an infinite-horizon MDP model, in which the 
objective is to minimize the expected discounted cost that captures overtime work, the waiting time, and the risk of adverse events.

We define type- $(i, j)$ patients as those who are given the urgency level $i \in$ $\{1,2, \ldots, I\}$ and have waited for $j \in\left\{0,1,2, \ldots, J_{i}\right\}$ periods on the waiting list, where $J_{i}$ is the maximum waiting time for a patient whose urgency level is $i$ and assumed to be non-decreasing in $i$. When a new patient joins the waiting list, an urgency level is initially given to the patient. The patient's urgency level remains the same regardless of the waiting time, but the patient type- $(i, j)$ changes over time in $j$. For example, a type- $(i, j)$ patient becomes a type- $(i, j+1)$ patient if the patient is not scheduled within a given period of time.

Let $x_{i j}^{t}$ be the number of type- $(i, j)$ patients waiting for surgery at the beginning of each period $t$ and define $s^{t}=\left\{x_{i j}^{t}: 1 \leq i \leq I, 0 \leq j \leq J_{i}\right\}$ as the vector of the number of patients on the waiting list. A set of patients is selected from $s^{t}$, and we define $u_{i j}^{t}$ as the number of type- $(i, j)$ patients selected for scheduling. Thus, $u^{t}=\left\{u_{i j}^{t}: 0 \leq u_{i j}^{t} \leq x_{i j}^{t}, 1 \leq i \leq I, 0 \leq j \leq J_{i}\right\}$ is the action for the state $s^{t}$.

Costs are incurred when a patient is not selected in the present decision period. Previous works have typically assumed a constant penalty without considering the urgency level for each patient and/or the amount of time the patient spent on the waiting list. Much research indicates that the waiting time may lead to the loss of opportunities for care, which can lead to higher costs from additional treatments and a lower quality of life, and to productivity loss (e.g., income loss). To address this issue, we introduce a unit period penalty $c_{w}(i, j)$ for the postponement of a type- $(i, j)$ patient. Thus, $c_{w}(i, j)$ is employed to represent the priority score for type- $(i, j)$ patients. When urgency levels are ordered from most urgent to least urgent, $c_{w}(i, j)$ is strictly decreasing in $i$ and increasing in $j$. However, it is not necessary to assume that it is linearly increasing in $i$ and $j$. This idea is consistent with the typical way in which previous studies have prioritized patients on the waiting list $[22,33]$.

Patients face the risk of leaving the waiting list before they are admitted for surgery, and this risk generally increases as the waiting time increases. We consider this risk by introducing the $\operatorname{cost} c_{r}$ arising when any type of patient leaves while waiting. The cost $c_{r}$ is separated from the postponement cost $c_{w}(i, j)$ rather than including it in the $c_{w}(i, j)$, because the patient who leaves the waiting list not only incurs a cost but also affects the waiting list (i.e., the state). To represent the number of patients leaving the waiting list, we introduce a binomial random variable $z_{i j}^{t}$ with parameters $\left(x_{i j}^{t}-u_{i j}^{t}\right)$ and $\alpha_{i j}$, where $\alpha_{i j}$ is the probability that a patient who is not admitted at time $t$ leaves the waiting list. Thus, $z_{i j}^{t}$ is represented as

$$
z_{i j}^{t} \sim B\left(x_{i j}^{t}-u_{i j}^{t}, \alpha_{i j}\right)=\left(\begin{array}{c}
x_{i j}^{t}-u_{i j}^{t} \\
k
\end{array}\right) \alpha_{i j}^{k}\left(1-\alpha_{i j}\right)^{x_{i j}^{t}-u_{i j}^{t}-k}
$$

$$
\text { where } k=0,1, \ldots, x_{i j}^{t}-u_{i j}^{t} \text {. }
$$

We assume that $\alpha_{i j}$ is strictly decreasing in $i$ and increasing in $j$. 
Let $\tau$ be the total surgery duration when the action $u^{t}$ is taken. Then $\tau$ is a random variable that follows a probability distribution denoted by $f^{u *}(\tau)$, where $f^{u *}(\tau)$ is defined by the $u$-fold convolution of an individual distribution based on the i.i.d.(independent and identically distributed) assumption. The cumulative distribution function (CDF) of $\tau$ is denoted by $F^{u *}(\tau)$. Although the surgery duration is dependent on surgery type, which may not reflect the real world, we assume that the distribution of individual patients' procedure time is independent and identical without sacrificing all the properties discussed in this work. Relaxing this assumption on identical surgery duration affects only the definition of $F^{u *}(\tau)$.

The surgical service group aims to schedule as many patients as possible to minimize costs associated with their postponements. However, overtime costs are incurred when the total surgery duration is over the regular capacity $B$, which is the total available time including every detail of surgery operations. Let $c_{o}(\tau)$ be the penalty for exceeding the regular capacity when the total surgery duration is $\tau$. Thus, the total expected overtime cost under the decision $u^{t}$ is given by $\int_{B}^{\infty} c_{o}(\tau)(\tau-B) \mathrm{d} F^{u *}(\tau)$. Further, let $c_{o}(\tau)$ take the following form when we define the maximum allowable overtime work as $(T-B)$ :

1. if $\tau \leq B$, then $c_{o}(\tau)=0$

2. if $\mathrm{B}<\tau<T$, then $c_{o}(\tau)>0, \frac{\partial}{\partial \tau} c_{o}(\tau) \geq 0$, and $\frac{\partial^{2}}{\partial \tau^{2}} c_{o}(\tau) \geq 0$ [10], and

3. if $\tau \geq T$, then $c_{o}(\tau)=M<\infty$, where $M$ is an arbitrary large number.

For a given state-action pair $\left(s^{t}, u^{t}\right)$, the present period expected cost function is written as follows:

$$
\begin{aligned}
\mathbb{E} c_{t}\left(s^{t}, u^{t}\right)= & \int_{B}^{\infty} c_{o}(\tau)(\tau-B) \mathrm{d} F^{u *}(\tau)+\sum_{i=1}^{I} \sum_{j=0}^{J_{i}}\left(1-\alpha_{i j}\right) c_{w}(i, j)\left(x_{i j}^{t}-u_{i j}^{t}\right) \\
& +\sum_{i=1}^{I} \sum_{j=0}^{J_{i}} c_{r} \alpha_{i j}\left(x_{i j}^{t}-u_{i j}^{t}\right) \\
= & \int_{B}^{\infty} c_{o}(\tau)(\tau-B) \mathrm{d} F^{u *}(\tau) \\
& +\sum_{i=1}^{I} \sum_{j=0}^{J_{i}}\left[\left(1-\alpha_{i j}\right) c_{w}(i, j)+\alpha_{i j} c_{r}\right]\left(x_{i j}^{t}-u_{i j}^{t}\right) .
\end{aligned}
$$

Note that the expected number of type- $(i, j)$ patients leaving the waiting list is given by the mean of the binomial distribution that is $\alpha_{i j}\left(x_{i j}^{t}-u_{i j}^{t}\right)$. Based on the assumption about $c_{o}(\tau)$ and the fact that $F^{(u+1) *}(\tau) \leq F^{u *}(\tau)$ for $\forall \tau \in \mathbb{R}_{+}$, we can show that $\mathbb{E} c(s, u)$ is piecewise-linear and convex in $u^{t}$ [21].

Now, we consider the arrival of new requests. Let $\mathrm{d}_{i j}^{t}$ be new demand requests of type- $(i, j)$ patients arrived in period $t$. We assume that $\left\{\mathrm{d}_{i j}^{t}: t=1,2, \ldots\right\}$ is a sequence of $i . i . d$. random variables following the Poisson distribution with parameter $\lambda_{i j}$. Because only new patients are added to the list, $\mathrm{d}_{i, j}^{t}=0$ unless $j=0$. 
The state transition is defined by $x_{i j+1}^{t+1}=x_{i j}^{t}-u_{i j}^{t}-z_{i j}^{t}+\mathrm{d}_{i j}^{t}$. Since $z_{i j}^{t}$ and $\mathrm{d}_{i j}^{t}$ are independent of each other, the transition probability is given as follows:

$$
P\left(s^{t+1} \mid s^{t}, u^{t}\right)=\prod_{i=1}^{I} \prod_{j=0}^{J_{i}} \sum_{k=0}^{x_{i j}^{t}-u_{i j}^{t}} P\left(z_{i j}^{t}=k\right) P\left(\mathrm{~d}_{i j}^{t}=x_{i j}^{t+1}-x_{i j}^{t}+u_{i j}^{t}+k\right) .
$$

Finally, for a given initial state $s, \phi_{u}(s)$ denotes the expected infinite horizon discounted cost under policy $u$. Then,

$$
\phi_{u}(s)=\lim _{T \rightarrow \infty} \mathbb{E}\left[\sum_{t=1}^{T} \gamma^{t-1} c_{t}\left(s^{t}, u^{t} \mid s\right)\right]
$$

where $\gamma(0 \leq \gamma<1)$ is the discount factor.

The optimal policy $u^{*}(s)$ is the one that minimizes the cost $\phi_{u}(s)$ starting in the initial state $s$. That is,

$$
\phi_{u^{*}}(s)=\min _{u \in U(s)} \phi_{u}(s)
$$

where $U(s)$ is a set of all admissible policies for the state $s$.

\section{Solution Procedure}

While MDP models are used in a wide range of applications, it is computationally too expensive to find an optimal policy within a reasonable amount of time. Therefore, Min and Yih [21] employed the structural properties of the optimal policy for designing computational algorithms to eliminate efforts to search non-optimal action and state spaces. The resulting decrease in the search space facilitates the procedure and improves computational efficiency, but the solution procedure also becomes ineffective when the size of the problem increases. To address this issue, we propose an iterative search procedure that provides an instance of an optimal solution in an online manner for a given initial state.

The underlying idea of the proposed iterative search procedure is to sequentially admit patients while the marginal cost is decreasing by adding a patient. To support this idea, we first show the convexity and monotonicity of a value function.

\subsection{Analysis of Structural Properties}

The total expected discounted cost in (2) leads to the following optimality equation with a finite-horizon $N$ :

$$
v_{n}\left(s^{n}\right)=\inf _{u^{n} \in U\left(s^{n}\right)} \mathbb{E}\left\{c_{n}\left(s^{n}, u^{n}\right)+\gamma v_{n+1}\left(s^{n+1}\right)\right\}, \text { for } n=1,2, \ldots, N-1,
$$

where $v_{n}\left(s^{n}\right)$ is a value function with state $s^{n}$, which denotes the minimum expected cost for the tail subproblem starting in period $n$ and ending in period $N$. Without loss of generality, we assume $v_{N}\left(s^{N}\right)=0$ for all $s^{N}$. 
To keep the notation simple, we drop the superscript or subscript of a vector in some cases. Let $J_{n}(s, u)=\mathbb{E}\left[c_{n}(s, u)+\gamma v_{n+1}(s-u-z+d)\right]$, so that $v_{n}(s)=$ $\min _{u \in U(s)} J_{n}(s, u)$.

Theorem 4.1. If $v_{N+1}(s)=0$ for all $s$, then $J_{n}(s, u)$ is jointly convex in $s$ and $u$, and $v_{n}(s)$ is convex in $s$ for all $n=1,2, \ldots, N$.

Proof. See Appendix

Lemma 4.2. The value function $v_{n}(s)$ is non-decreasing in $x_{i, j} \in s$ for all $i, j$.

Proof. Proof is given by induction. For $n=N-1$, because the single-period cost function $\mathbb{E} c(s, u)$ given in (1) is non-decreasing in $x_{i j} \in s$ and $v_{N}(s)=0, v_{N-1}(s)$ becomes non-decreasing in $x_{i j} \in s$. If the property is assumed to be hold for $n=N-2$, we can conclude that $v_{N-3}(s)$ is non-decreasing in $x_{i j} \in s$.

In the infinite horizon case, $v(s)=\lim _{n \rightarrow \infty} v_{n}(s)$ and the resulting stationary policy is also optimal [2]. Thus, the convexity holds for the infinite horizon model as well. The convexity and monotonicity of the value function shown in Theorem 1 and Lemma 1 lead to the lower bound of the optimal infinite-horizon solution. Providing a lower bound eliminates the need for evaluating any infeasible solutions below the lower bound. Proposition 1 proves that the single-period solution is the lower bound.

Proposition 4.3. For any state s, the optimal number of patients selected from the waiting list is more than the optimal single-period solution. That is,

$$
u_{i j}^{0} \leq u_{i j}^{*} \text { for all } i, j \text { and therefore }\left\|u^{0}\right\|_{1} \leq\left\|u^{*}\right\|_{1},
$$

where $u_{i j}^{0} \in u^{0}$ and $u_{i j}^{*} \in u^{*}$ represent the optimal single-period solution and the optimal infinite-horizon solution for the state $s$, respectively.

Proof. Let $\Delta_{i j}$ be a unit vector for which the $(i, j)$-th element is one and the others are zero. Then, because $u^{0}$ is an optimal solution for $\mathbb{E}[c(s, u)]$, the following inequality holds:

$$
\mathbb{E}\left[c\left(s, u^{0}\right)-c\left(s, u^{0}-\Delta_{i j}\right)\right] \leq 0 .
$$

Now, we assume $u_{i j}^{0}=u_{i j}^{*}+1$ for $\exists i, j$ and $\left\|u^{0}\right\|_{1}=\left\|u^{*}\right\|_{1}+1$, which means that the optimal infinite horizon solution is less than $u^{0}$, and then the following inequality holds:

$$
\begin{aligned}
& J\left(s, u^{0}\right)-J\left(s, u^{0}-\Delta_{i j}\right)>0, \text { for } \exists i, j \\
\Leftrightarrow & \int_{B}^{\infty} c_{o}(\tau)(\tau-B) \\
& \times \mathrm{d} F^{u *}(\tau)-\int_{B}^{\infty} c_{o}(\tau)(\tau-B) \mathrm{d} F^{(u-1) *}(\tau)-\left[\left(1-\alpha_{i j} c_{w}(i, j)+\alpha_{i j} c_{r}\right]\right. \\
& +\gamma \mathbb{E}\left[v\left(s-u^{0}-z+d\right)-v\left(s-u^{0}+\Delta_{i j}-z^{\prime}+d\right)\right]>0 \\
\Leftrightarrow & \mathbb{E}\left[c\left(s, u^{0}\right)-c\left(s, u^{0}-\Delta_{i j}\right)\right] \\
& +\gamma \mathbb{E}\left[v\left(s-u^{0}-z+d\right)-v\left(s-u^{0}+\Delta_{i j}-z^{\prime}+d\right)\right]>0,
\end{aligned}
$$


where $z \sim B\left(s-u^{0}, \alpha\right)$ and $z^{\prime} \sim B\left(s-u^{0}+\Delta_{i j}, \alpha\right)$.

However, $v\left(s-u^{0}-z+d\right)-v\left(s-u^{0}+\Delta_{i j}-z^{\prime}+d\right) \leq 0$ by Lemma 1 , and $\mathbb{E}\left[c\left(s, u^{0}\right)-c\left(s, u^{0}-\Delta_{i j}\right)\right] \leq 0$. Thus, $J\left(s, u^{0}\right)-J\left(s, u^{0}-\Delta_{i j}\right) \leq 0$ which conflicts with the assumption $u_{i j}^{0}=u_{i j}^{*}+1$.

In contrast to the clear identification of the minimum number of patients selected from the waiting list, the upper bound is not easy to be found. While the precise placement of the upper bound is unclear, the intuition based on the capacitated operating room model supports the existence of an upper bound. Because the maximum overtime work is given as $T-B$, the schedule accepts no more than the number of patients for which the total surgery duration is over $T$.

The following Proposition 2 shows the structure of an optimal policy. The first part of Proposition 2 presents the monotone property of the optimal policy, which states that more patients should be admitted if there are more patients on the waiting list. The remaining of Proposition 2 regulates the number of patients added to the operating room schedule and specifies an optimal policy for managing the waiting list. The second part of Proposition 2 describes that at most one patient will be admitted when the size of the waiting list increases by 1 . The intuition behind the last part is that the decision relies not only on the overall demand, and the decision may differ from others whose state has the same cardinality. In other words, if we have more patients who incur higher cost while the size of surgery waiting list remains the same, then more patients will be added to the operating room schedule.

Proposition 4.4. Let $u^{*}(s)$ be the optimal policy for a state s. Then, the following statements hold:

1. The optimal policy $u^{*}(s)$ is non-decreasing in $s$. That $i s, u_{i j}^{*} \leq u_{i j}^{\prime}$ for all $i, j$, where $u_{i j}^{*} \in u^{*}(s)$ and $u_{i j}^{\prime} \in u^{*}\left(s+\Delta_{i j}\right)$.

2. If $x_{i j}$ increases by 1 , then the optimal decision for the type- $(i, j)$ patient $u_{i j}^{*}$ increases by at most 1. $u_{i j}^{*} \leq u_{i j}^{\prime} \leq u_{i j}^{*}+1$, where $u_{i j}^{*} \in u^{*}(s)$ and $u_{i j}^{\prime} \in$ $u^{*}\left(s+\Delta_{i j}\right)$.

3. For a state $s, u_{k l}^{*} \geq u_{k l}^{\prime}$ for all $k, l$, where $u_{k l}^{\prime} \in u\left(s+\Delta_{m n}\right)$ and $u_{k l}^{*} \in u\left(s+\Delta_{i j}\right)$ if $\left(1-\alpha_{i j}\right) c_{w}(i, j)+c_{r} \alpha_{i j} \geq\left(1-\alpha_{m n}\right) c_{w}(m, n)+c_{r} \alpha_{m n}$.

Proof. See Appendix.

\subsection{Approximation-BAsed iterative SEARCh PROCEDURE}

The proposed iterative search procedure starts from the optimal solution of a single-period model (i.e., the lower bound shown in Proposition 1). Because $J(s, u)$ is convex in $u$, one additional patient of type- $(i, j)$ added to the operating room schedule reduces the expected marginal gain presented by $\frac{\partial}{\partial u_{i j}} J(s, u)=J(s, u)-$ $J\left(s, u+\Delta_{i j}\right)$ as a result of increases in the expected overtime cost and decreases in the expected costs associated with the waiting time. Thus, the procedure keeps 
adding patients to the schedule as long as the expected marginal gain is nonnegative.

The most challenging part of the procedure is that it is not trivial to determine both $\frac{\partial}{\partial u_{i j}} J(s, u)$ and $\Delta_{i j}$ (i.e., the type of patient that should be the next to be selected). Because the value function $v(s)$ that is unknown for all states $s$, but which should be available for the calculation of the marginal gain. To address this issue, we approximate the value function by employing a sampling-based finite-horizon approximation method, not by seeking true value functions. This approximation procedure is referred to as the "Rollout procedure" [3,5,14,26]. An example of this approximation method in the healthcare field can be found in Thompson et al. [34].

In each period, a set of random samples are used to estimate the value function, and a better policy is selected based on the estimation. Formally, suppose that we estimate $J(s, u)$ for the current state $s$ by using the sample average $\hat{J}_{N}(s, u)$ for the finite horizon $N$, then

$$
\hat{J}_{N}(s, u)=\mathbb{E} c(s, u)+\gamma \frac{1}{M} \sum_{m=1}^{M} \hat{Q}_{N-1}^{*}\left(s_{m}\right),
$$

and $\hat{Q}_{n}^{*}(s)$ is defined by

$$
\hat{Q}_{n}^{*}(s)=\inf _{u \in U(s)}\left\{\mathbb{E} c(s, u)+\gamma \frac{1}{M} \sum_{m=1}^{M} \hat{Q}_{n-1}^{*}\left(s_{m}\right)\right\},
$$

where $n=1,2, \ldots, N, M$ is the sample size, $\hat{Q}_{0}^{*}(s)=0$ for all $s$, and $s_{m}$ is the next state simulated by the sample $m$.

The solution given by Equation (4) is a nonstationary stochastic policy [14]. Moreover, the resulting stochastic policy is interpreted as an approximate receding $N$-horizon control for the infinite horizon model in the context of the "rolling horizon procedure" [12]. This implies that the optimal solution for the infinite horizon model can be approximated by solving a sufficiently long finite horizon problem. A number of studies examining the rolling horizon procedure have indicated that both the optimal solution and costs for finite horizon approximation converge to the true optimal infinite horizon solution and costs as the horizon approaches to infinity $[4,27]$.

Now we are ready to present the iterative search procedure. This procedure iteratively allocates patients into the operating room schedule until the estimated marginal gain becomes negative. The final procedure is given as follows:

\section{Iterative Search Procedure: Sampling-based N-horizon Approxima- tion}

1. Initialize

(a) Obtain the optimal single-period solution $u^{0}\left(s_{0}\right)$, where $s_{0}$ is the initial state.

(b) Let $u$ be the current solution and set $u=u^{0}\left(s_{0}\right)$. Set $s_{r}=s_{0}-u$. 
2. Calculate the expected marginal gain

(a) If $s_{r}$ is not equal to the vector of all zeros, then continue. Otherwise, go to Step 4.

(b) Estimate the marginal gain $\frac{\partial}{\partial u_{i j}} \hat{J}\left(s_{0}, u\right)=\hat{J}\left(s_{0}, u\right)-\hat{J}\left(s_{0}, u+\Delta_{i j}\right)$ for each $i \in\{1,2, \ldots, I\}$ and $j=$ $\max \left\{j: x_{i j}^{r}>0, j \in\left\{0,1, \ldots, J_{i}\right\}\right\}$, where $x_{i j}^{r}$ is a set of elements in $s_{r}$.

(c) Set $\Delta_{i j}^{*}=\arg \max _{i} \frac{\partial}{\partial u_{i j}} \hat{J}\left(s_{0}, u\right)$.

3. Evaluate the estimated marginal gain

(a) If $\frac{\partial}{\partial u_{i j}} \hat{J}\left(s_{0}, u\right) \geq 0$, then $u \leftarrow u+\Delta_{i, j}^{*}, s_{r} \leftarrow s_{r}-\Delta_{i j}^{*}$ and go to Step 2 . Otherwise, go to Step 4.

4. Stop the iteration, and $u$ is the final solution.

\section{Evaluation of AN OPtimal POLiCy}

This section summarizes the results of numerical experiments that we conduct to show an optimal policy and to evaluate the effects of uncertainty considered in the model.

\subsection{Estimating MOdel PARAmeters}

The test problem is constructed by using the data from the literature [30,35] and by considering an actual regional hospital with some reasonable modifications. For simplicity, we consider only the case of two urgency levels: urgent and non-urgent (i.e., $I=2$ ).

Because of the lack of actual data, we arbitrarily choose all the cost parameters $\left(e . g ., c_{w}(i, j), c_{r}\right.$, and $\left.c_{o}(\tau)\right)$, but make some reasonable modifications. $c_{w}(i, 0)$ indicates an urgency level initially given to each patient, and we estimate the ratio of $c_{w}(1,0)$ to $c_{w}(2,0)$ from the data on the number of patients selected from the waiting list per week because the decision maker expedites those surgical procedures that incur higher costs. According to the data on elective CABG surgery from Sobolev and Kuramoto [30], the weekly number of urgent patients admitted is approximately three times greater than that for non-urgent patients. For other surgery types, the ratio of weekly admission for the most urgent patients to the least urgent patients is three to six. In this study, we set $c_{w}(1,0)$ to be three times greater than $c_{w}(2,0)$, and arbitrarily set $c_{w}(2,0)$ as 2 . Furthermore, we assume $c_{w}(i, j)$ to be linearly increasing in $j$ with the coefficient $w$, and we vary a coefficient $w$ from 0 to 7 . Thus, $c_{w}(i, j)$ is written by:

$$
c_{w}(i, j)=(6-(i-1) \times 3)+w \times j, \quad \text { where } w \in \mathbb{R}_{+} .
$$

Intuitively the cost $c_{r}$ incurred as a result of emergency surgery or death of a patient is at least greater than the total expected cost of the patient remaining in the 
waiting list. Under this intuition, $c_{r}$ is estimated by its relationship with $c_{w}(i, j)$ :

$$
\begin{aligned}
\alpha_{i j} c_{r} & \geq\left(1-\alpha_{i j}\right) c_{w}(i, j)+\left(1-\alpha_{i j+1}\right)^{2} c_{w}(i, j+1)+\cdots+\left(1-\alpha_{i J_{i}}\right)^{J_{i}-j} c_{w}\left(i, J_{i}\right) \\
& \geq\left(1-\alpha_{i j}\right) c_{w}(i, j)+\left(1-\alpha_{i j}\right)^{2} c_{w}(i, j)+\cdots+\left(1-\alpha_{i j}\right)^{J_{i}-j} c_{w}(i, j) \\
& =\frac{1-\alpha_{i j}}{\alpha_{i j}}\left(1-\left(1-\alpha_{i j}\right)^{J_{i}-j}\right) c_{w}(i, j) .
\end{aligned}
$$

Here $J_{i}$ is derived from the estimated probability that a CABG surgery patient remains on the waiting list. According to the data in Sobolev and Kuramoto [30], in the case of patients with urgent status, the probability is approximately zero when the waiting time was about 40 weeks. By contrast, the probability for nonurgent patients is approximately $90 \%$ at the waiting time of 50 weeks. Thus, based on the estimated probability, we roughly decide that $J_{1}=40$ and $J_{2}=50$.

Because the cumulative risk of becoming emergency surgery or death increases almost linearly with the waiting times [30], $\alpha_{i j}$ is defined as $\alpha_{i j}=\frac{1}{a_{i}} \times j$, where $a_{i}$ is the unit increment of the probability. In the case of elective cholecystectomy surgery, although the probability of emergency surgery is low during the first 4 weeks (approximately 2\%), it increases dramatically to $40 \%$ when the waiting time is 40-52 weeks. In terms of CABG surgery, approximately $10 \%$ of urgent patients and only $4 \%$ of non-urgent patients undergo emergency surgery or die when the waiting time is over 50 weeks. We vary $a_{i}$ from 20 to 300 (i.e., $\alpha_{i j}$ is between $0.003 \times j$ and $0.05 \times j$ ) and investigate the effects of the risk associated with leaving the waiting list.

We set the regular operating room capacity at $780 \mathrm{~min}$ for each period (i.e., $B=780 \mathrm{~min}$ ), and the maximum overtime is arbitrarily given as $180 \mathrm{~min}$ (i.e., $T-B=180 \mathrm{~min})$. Overtime work is allowed with the overtime costs $c_{o}(\tau)$ when the total surgery duration is $\tau . c_{o}(\tau)$ is defined as $t_{k} \times \tau$, where $t_{k}$ is the coefficient of the unit overtime cost that doubles every 30 min during overtime. The subscript $k$ denotes different levels of the capacity constraint. We define three levels of the capacity constraints. The initial value of $t_{k}$ is $0.25,0.4$ and 0.8 , respectively. Note that $c_{o}(\tau)=\infty$ when $\tau$ reaches at the maximum allowable overtime.

Instead of assuming a particular distribution, the distribution of surgery duration is derived from actual data of surgery procedures that we compiled over 3700 cases during a seven-month period. While the surgery duration depends on surgery type and the patient's condition, we assume that all the patients in the same surgical service group follow the same distribution. For instance, according to the data on the distribution of urology surgery duration, the mean is $64 \mathrm{~min}$, the standard deviation is $52 \mathrm{~min}$ and the skewness is 2.11. A set of surgery durations is randomly selected from the distribution of urology surgery durations to calculate the expected overtime.

We determine the arrival of new patients by using data from Vasilakis et al. [35]. Their simulation method models new arrivals as having a Poisson distributions with parameters 6 and 7 for urgent and non-urgent levels, respectively. 
TABLE 1. Convergence of the objective value $\left(\phi^{*}\right)$ with different values of $N$.

\begin{tabular}{lcccccccc}
\hline$N$ & 1 & 2 & 3 & 4 & 5 & 6 & 7 & 8 \\
\hline$\phi^{*}$ & 2616.4 & 7202.8 & 10394.8 & 12850.8 & 14479.3 & 15372.1 & 15717.4 & 15892.4 \\
$\Delta \phi^{*}$ & - & 4586.4 & 3192.0 & 2456.0 & 1628.5 & 892.8 & 345.3 & 175.0 \\
$\Delta \phi^{*}(\%)$ & - & 40.9 & 22.2 & 14.6 & 8.8 & 4.6 & 1.8 & 0.9 \\
\hline
\end{tabular}

\subsection{The BeHAVior of The ITERATIVE SEARCH PROCEDURE}

This section verifies the theoretical results established in Section 4. We first run numerical experiments with different values of $N$ to determine the appropriate value by observing the convergence of the objective value. Table 1 shows that the average objective value stabilizes over 5 replications with 10 samples at each stage (i.e., $M=10$ ) when $N$ is expanded to 8 periods. We perform 10 replications for each problem by using different streams of random numbers and use the same set of seeds for the random number generation for other experiments (i.e., common random numbers are used).

Solution quality and computation time are sensitive to the values of $M$ and $N$, and thus those two elements require close attention. For a fixed value of $N$, a large sample of $M$ reduces the solution variance [5], and we have shown that a larger $N$ better approximates the true cost. According to the computational experiments, $M$ over 10 provides stable outcomes for any given $N$. The average computational time is about $30 \mathrm{~s}$ per iteration for $N=6$ and increases exponentially with $N$. Thus, we use $N=5$ for further experiments despite better solution quality with a lager value of $N$.

With $N=5$, it takes, on average, less than $10 \mathrm{~s}$ per iteration. An optimal single-period solution is found within one second regardless of the problems size that is primarily defined by the number of patients on the waiting list. Because this procedure generally iterates less than 10 times, the total computational times is not over $90 \mathrm{~s}$. Although the solution procedure proposed by Min and Yih [21] outperforms the conventional value iteration procedure, it takes approximately $500 \mathrm{~s}$ to obtain a policy in the case of 20 patients on the waiting list. Note that problem size has no substantial effect on the proposed iterative search procedure. This indicates that the proposed procedure may be particularly effective for large problems.

Noteworthy is the convexity of $J(s, u)$. Figure 1 plots the estimated marginal gain from adding patients to the operating room schedule. The results of the numerical experiments summarized in Section 5 indicate that with each iteration, the iterative search procedure improves the solution based on this convexity. The estimated marginal gain becomes negative when 8 more patients are added to the single-period optimal solution. Thus, the final solution is determined by adding 7 patients to the single-period optimal solution. 


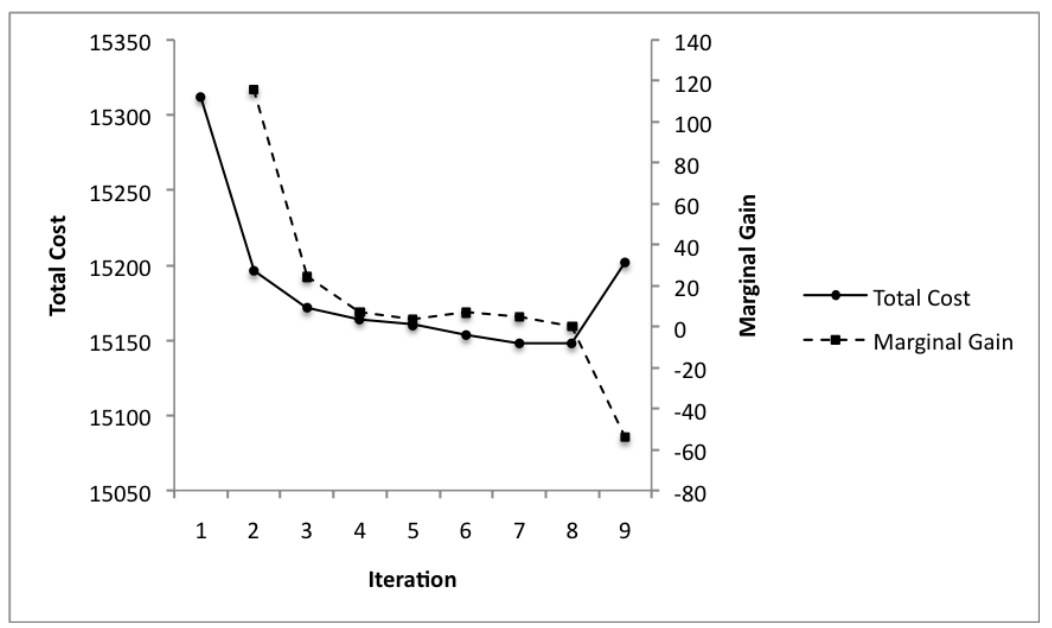

FiguRE 1. Monotone decrease of marginal gain.

The results of each iteration indicate that the total cost is strictly decreasing before the final solution. However, it is not necessarily true that the estimated marginal gain also strictly decreases at each iteration. A nonstationary stochastic policy due to the sampling-based approximation yields the non-monotone behaviors.

\subsection{THE POLICY}

This section introduces an optimal policy obtained by using the iterative search procedures presented in Section 4.2. Recall that the major interest in this work is to determine the number of patients admitted for surgery from the waiting list.

To provide a better understanding of the optimal policy, we consider a special case in which $c_{w}(i, j)$ is constant in $j$ and $\alpha_{i j}=0$ for all $i$ and $j$, that is, a case of the static-priority model. Figure 2 illustrates an example of optimal policy obtained by the modified value iteration method of which a detailed description is found in Min and Yih [21]. Each point indicates the total number of patients selected from the waiting list corresponding to the total number of patients waiting. The solid line is the number of admitted patients determined from the model that does not discriminate between different patient priorities. The number of admitted patients varies not only by the number of patients waiting for surgery but also by that belonging to each patient type. As the number of urgent patients on the waiting list increased, the policy tends to allocate more patients to the capacity.

\subsection{EFFECTS OF TIME-DEPENDENT PRIORITY}

In this section, we assess the effects of the time-dependent patient priority by conducting simple simulation experiments that process patients according to the 


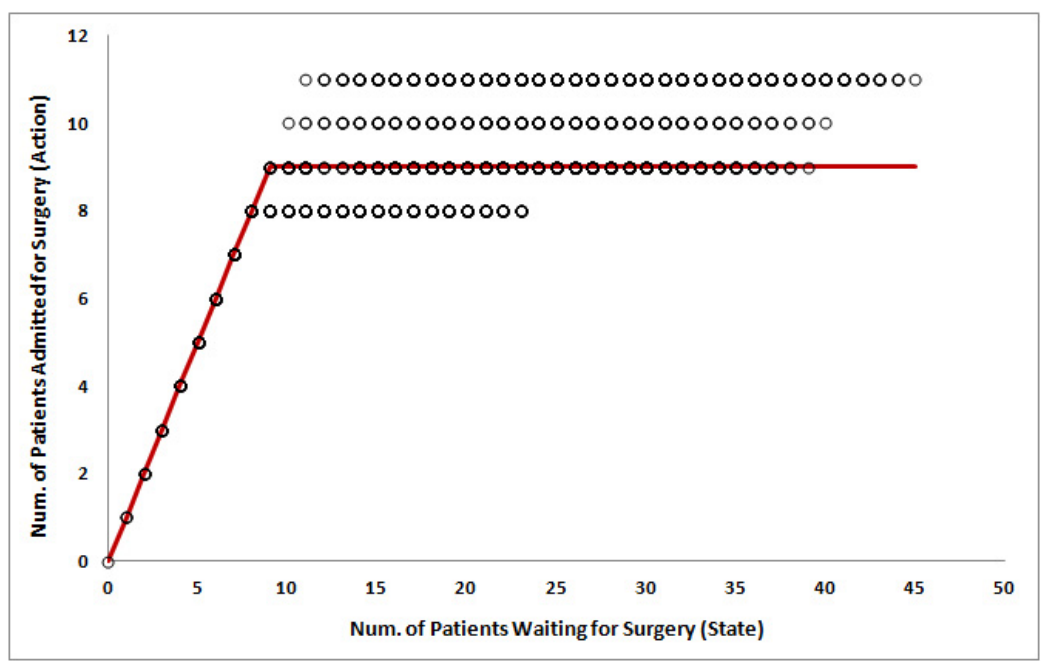

Figure 2. Optimal Policy: the static-priority model, $c_{w}(1, j)=$ $240, c_{w}(2, j)=80, c_{w}(3, j)=40$.

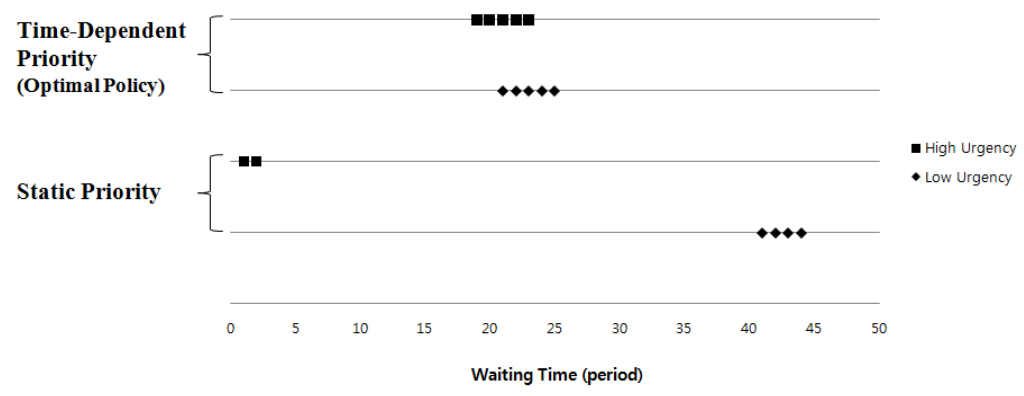

FiguRE 3. Effects of the time-dependent priority on the waiting time.

solution obtained through the proposed procedure. As shown in Figure 3, the time-dependent priority (i.e., $c_{w}(i, j)<c_{w}(i, j+1)$ ) reduces the waiting time for less urgent patients while increasing it for more urgent patients. Considering the waiting time in determining priority allows less urgent patients to occupy the operating room capacity earlier to prevent cost associated with the waiting time from increasing. In the case of the static-priority setting $\left(i . e ., c_{w}(i, j)=c_{w}(i, j+1)\right)$, the waiting time for less urgent patients increases substantially because patients with higher urgency level are always admitted first and only the remaining capacity is given to patients with lower urgency level. 
Figure 4 illustrates the behavior of a policy to different levels of $a_{i}$ (the coefficient for $\alpha_{i j}$ representing the risk of adverse events) defined in Section 5.1. The policy exhibits a convex shape (i.e., the number of patients admitted for surgery is initially decreasing and then increasing) in $\alpha_{i j}$. A small $a_{i}$ value (i.e., a high probability that a patient leaves the waiting list while waiting) shortens the waiting list, but there are substantial increases in costs associated with their departure. These cost increases allow the model to allocate more patients into the operating room schedule. In addition, a large probability removes many patients from the waiting list with constant $\operatorname{cost} c_{r}$, which makes the marginal increment of the total cost small when the probability becomes fairly large. On the contrary, when $a_{i}$ is relatively large, only a small number of patients are expected to leave the waiting list, increasing the waiting time and eventually leading to higher costs in the future. Therefore, patients are slightly more likely to be admitted for surgery when the risk of patients leaving the waiting list is small.

The solutions for different $a_{i}$ values indicates that the number of patients selected from each urgency level $u_{i}$ depends on the probability $\alpha_{i j}$. For example, $\left(u_{1}, u_{2}\right)=(7.0,3.6)$ for $\left(a_{1}, a_{2}\right)=(60,120)$ on average. The solution becomes $\left(u_{1}, u_{2}\right)=(4.6,6.6)$ when $a_{1}=120$ while $a_{2}$ remains as 120 .

As shown in Figure 4, which shows different levels of the capacity constraints, a strong capacity constraint mitigates the effects of $\alpha_{i j}$ implying that under a strong capacity constraint, there is little room for overtime work and thus for allocating more patients. When $t_{k}=0.8$, the solution is almost independent of the probability. It implies that a strong capacity constraint weakens the effects of considering the time-dependent patient priority.

\section{Conclusion}

This work proposes a model for managing a surgery waiting list that takes into account the dynamic patient status, which is formulated as the time-dependent cost of surgery postponements and the probability of patients leaving the waiting list, to select an appropriate number of patients from the waiting list. The decision attempts to minimize an expected infinite horizon discounted cost that captures overtime work, the waiting time, and the risk of adverse events.

There are several possible extensions to our model. Although the results indicate that the proposed procedure is computationally efficient, there is a need for an analysis of the complexity of the proposed algorithms. In line of this research interest, the iterative search procedure evaluates only one patient at each iteration (i.e., single-step size). Thus, further research should develop a procedure in which multiple patients are allocated at each iteration (i.e., multiple-step size). A procedure reflecting the multiple-step size should significantly reduce the computation time, but the step size needs to be carefully determined since a long step-size over the final solution may increase the number of iterations.

Another potential route of extension is to use previously acquired data for estimating the marginal gain, $\frac{\partial}{\partial u_{i j}} J(s, u)$. The software application can easily keep 


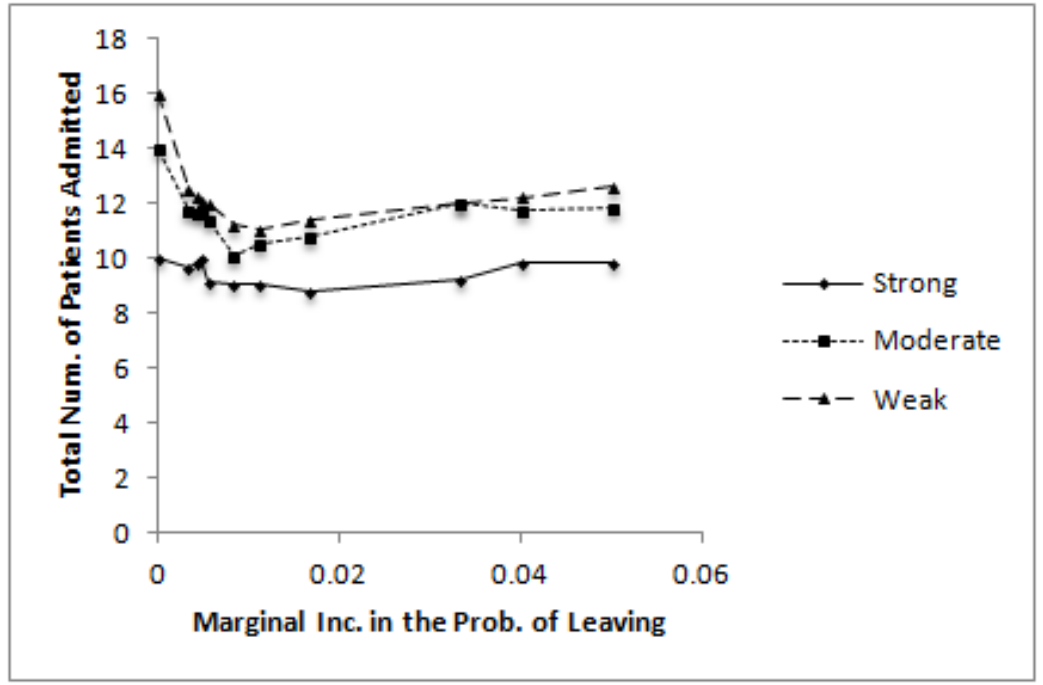

(a) Total num. of patients Admitted

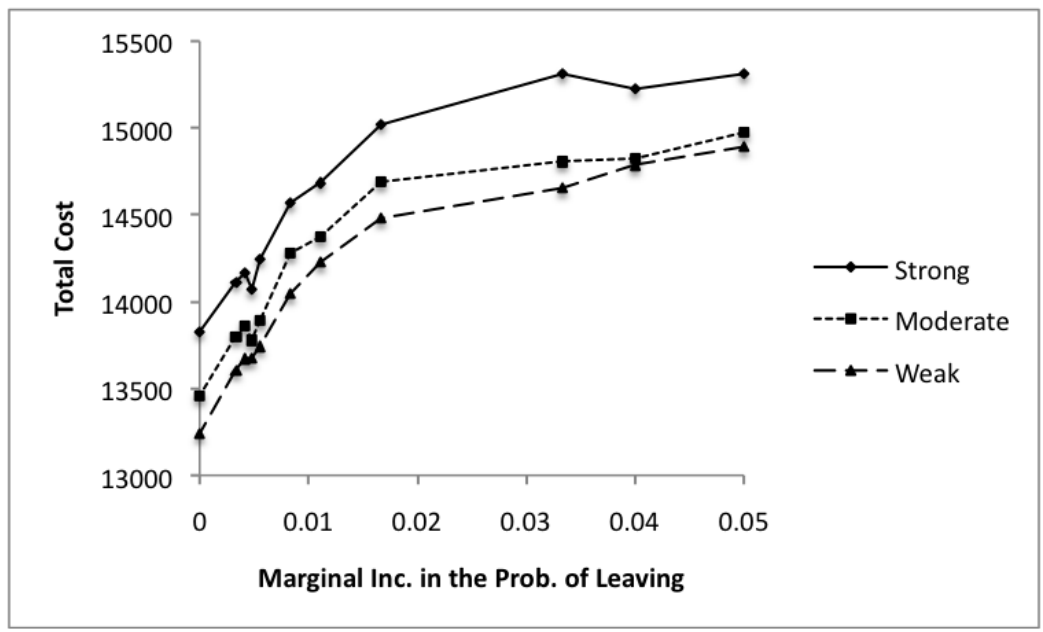

(b) Total cost

FiguRE 4. Effects of $\alpha_{i j}$ and capacity constraints.

track of all resulting data elements, including $\hat{Q}_{n}^{*}(s)$ in equation (4) and corresponding solutions. In general, the procedure can utilize a large amount of data that specifies how the function $\hat{Q}_{n}^{*}(s)$ is implicitly defined for the next procedure, and it will remove the amount of efforts needed for evaluating marginal gains. In this sense, the idea is extensible to another procedure that separates the training phase for approximating true value functions from the acting phase that runs the 
iterative search procedure without estimating marginal gains. It is unclear how the value function could be approximated, but Powell [26] described several methods for approximating value functions, which are generally referred to as approximate dynamic programming (ADP).

\section{APPENDIX}

\section{Proof of Theorem 4.1}

Proposition 4.3. The single-period cost function $\mathbb{E}[c(s, u)]$ is jointly convex in $s$ and $u$.

Proof. The function $\int_{T}^{\infty} c_{o}(\tau)(\tau-B) \mathrm{d} F^{u *}(\tau)$ is convex and increasing in $\tau$ for all $B \geq 0[8]$. Note that the total amount of service time $\tau$ is strictly increasing $u$. Because $c(s, u)$ is the sum of two convex functions, $c(s, u)$ is also convex in $u$. Furthermore, $\mathbb{E}[c(s, u)]$ is convex in $x_{i, j} \in s$.

Theorem 4.1. If $v_{N+1}(s)=0$ for all s, then, $J_{n}(s, a)$ is jointly convex in $s$ and $u$ and $v_{n}(s)$ is convex in $s$ for all $n=1,2, \ldots, N$.

Proof. The proof is given by induction on $n$. Here $v_{N+1}(s)$ and $J_{N}(s, u)$ are convex by assumption and Proposition 4.3. Assuming that the results hold for $n, v_{n}(s)$ and $J_{n}(s, u)$ are convex. We now show that the results hold also for $n-1$. Because both $v_{n}(s)$ and $c(s, u)$ are convex, $J_{n-1}(s, u)$ is also convex. The convexity of $J_{n-1}(s, u)$ supports that $v_{n-1}(s)$ is convex.

\section{Proof of Proposition 4.4}

Proof. 1. To keep notation simple, instead of $u^{*}(s)$, let $u$ denote an optimal solution for a state $s$. If we assume $u_{m n}^{*}>u_{m n}^{\prime}$ for $\exists m, n$, then the following inequality holds:

$$
\begin{aligned}
& J\left(s+\Delta_{i j}, u\right)-J\left(s+\Delta_{i j}, u-\Delta_{m n}\right) \\
= & \int_{B}^{\infty} c_{o}(\tau)(\tau-B) \mathrm{d} F^{u *}(\tau) \\
& -\int_{B}^{\infty} c_{o}(\tau)(\tau-B) \mathrm{d} F^{(u-1) *}(\tau)-\left(1-\alpha_{m n}\right) c_{w}(m, n)-\alpha_{m n} c_{r} \\
& +\gamma \mathbb{E}\left[v\left(s+\Delta_{i j}-u-B\left(s+\Delta_{i j}-u, \alpha\right)+d\right)\right. \\
& \left.-v\left(s-u+\Delta_{i j}+\Delta_{m n}-B\left(s-u+\Delta_{i j}+\Delta_{m n}, \alpha\right)+d\right)\right] \geq 0 .
\end{aligned}
$$


By the convexity and monotonicity of the value function,

$$
\begin{aligned}
& J\left(s+\Delta_{i j}, u\right)-J\left(s+\Delta_{i j}, u-\Delta_{m n}\right) \\
& <\int_{B}^{\infty} c_{o}(\tau)(\tau-B) \mathrm{d} F^{u *}(\tau) \\
& -\int_{B}^{\infty} c_{o}(\tau)(\tau-B) \mathrm{d} F^{(u-1) *}(\tau)-\left(1-\alpha_{m n}\right) c_{w}(m, n)-\alpha_{m n} c_{r} \\
& +\gamma \mathbb{E}\left[v\left(s-u+\Delta_{i j}-B\left(s-u+\Delta_{i j}, \alpha\right)+d\right)-v\left(s-u+2 \Delta_{i j}\right.\right. \\
& \left.\left.-B\left(s-u+2 \Delta_{i j}, \alpha\right)+d\right)\right] \\
& <\int_{B}^{\infty} c_{o}(\tau)(\tau-B) \mathrm{d} F^{u *}(\tau)-\int_{B}^{\infty} c_{o}(\tau)(\tau-B) \mathrm{d} F^{(u-1) *}(\tau) \\
& -\left(1-\alpha_{i j}\right) c_{w}(i, j)-\alpha_{i j} c_{r} \\
& +\gamma \mathbb{E}\left[v(s-u-B(s-u, \alpha)+d)-v\left(s-u+\Delta_{i j}-B\left(s-u+\Delta_{i j}, \alpha\right)+d\right)\right] \\
& =J(s, u)-J\left(s, u-\Delta_{i j}\right) .
\end{aligned}
$$

Because $u$ is an optimal solution for the state $s$ and minimizes $J(s, u), J(s, u)-$ $J\left(s, u-\Delta_{i j}\right) \leq 0$. Then $J\left(s+\Delta_{i j}, u^{*}(s)\right)-J\left(s+\Delta_{i j}, u^{*}(s)-\Delta_{m n}\right)<J(s, u)-$ $J\left(s, u-\Delta_{i j}\right) \leq 0$. It contradicts to the assumption $J\left(s+\Delta_{i j}, u^{*}(s)\right)-J(s+$ $\left.\Delta_{i j}, u^{*}(s)-\Delta_{m n}\right) \geq 0$ representing $u_{m n}^{*}>u_{m n}^{\prime}$. In a concluding manner, $u_{m n}^{*} \leq u_{m n}^{\prime}$ for all $m, n$, where $u_{m n}^{*} \in u^{*}(s)$ and $u_{m n}^{\prime} \in u^{*}\left(s+\Delta_{m n}\right)$.

2. Because $u$ is an optimal solution for the state $s$, the following inequality holds:

$$
\begin{aligned}
& J\left(s, u+\Delta_{i j}\right)-J(s, u) \geq 0 \\
& \Leftrightarrow \int_{B}^{\infty} c_{o}(\tau)(\tau-B) \mathrm{d} F^{(u+1) *}(\tau) \\
& -\int_{B}^{\infty} c_{o}(\tau)(\tau-B) \mathrm{d} F^{u *}(\tau)-\left(1-\alpha_{i j}\right) c_{w}(i, j)-\alpha_{i j} c_{r} \\
& +\gamma \mathbb{E}\left[v\left(s-u-\Delta_{i j}-B\left(s-u-\Delta_{i j}, \alpha\right)+d\right)\right. \\
& -v(s-u-B(s-u, \alpha)+d)] \geq 0 .
\end{aligned}
$$

Assume that $u_{i j}$ is increased by 2 while $x_{i j}$ is increased by 1 .

$$
\begin{aligned}
& J\left(s+\Delta_{i j}, u+2 \Delta_{i j}\right)-J\left(s+\Delta_{i j}, u+\Delta_{i j}\right) \\
& =\int_{B}^{\infty} c_{o}(\tau)(\tau-B) \mathrm{d} F^{(u+2) *}(\tau)-\int_{B}^{\infty} c_{o}(\tau)(\tau-B) \mathrm{d} F^{(u+1) *}(\tau) \\
& -\left(1-\alpha_{i j}\right) c_{w}(i, j)-\alpha_{i j} c_{r} \\
& +\gamma \mathbb{E}\left[v\left(s-u-\Delta_{i j}-B\left(s-u-\Delta_{i j}, \alpha\right)+d\right)\right. \\
& -v(s-u-B(s-u, \alpha)+d)] \\
& \geq \int_{B}^{\infty} c_{o}(\tau)(\tau-B) \mathrm{d} F^{(u+1) *}(\tau) \\
& -\int_{B}^{\infty} c_{o}(\tau)(\tau-B) \mathrm{d} F^{u *}(\tau)-\left(1-\alpha_{i j}\right) c_{w}(i, j)-\alpha_{i j} c_{r}
\end{aligned}
$$




$$
\begin{aligned}
& +\gamma \mathbb{E}\left[v\left(s-u-\Delta_{i j}-B\left(s-u-\Delta_{i j}, \alpha\right)+d\right)\right. \\
& -v(s-u-B(s-u, \alpha)+d)] \\
& =J\left(s, u+\Delta_{i j}\right)-J(s, u) \geq 0 .
\end{aligned}
$$

Thus, $J\left(s+\Delta_{i j}, u+2 \Delta_{i j}\right) \geq J\left(s+\Delta_{i j}, u+\Delta_{i j}\right)$ and it means that $u+2 \Delta_{i j}$ cannot be an optimal solution for the state $s+\Delta_{i j}$.

3. Let $J^{1}$ be a function corresponding to the state $s+\Delta_{i j}$ so that $u^{*}\left(s+\Delta_{i j}\right)=$ $\arg \min _{u \in U\left(s+\Delta_{i j}\right)} J^{1}\left(s+\Delta_{i j}, u\right)$. Similarly, let $J^{2}$ be a function for the state $s+\Delta_{m n}$ and it satisfies $u^{*}\left(s+\Delta_{m n}\right)=\arg \min _{u \in U\left(s+\Delta_{m n}\right)} J^{2}\left(s+\Delta_{m n}, u\right)$.

Because $u^{*}\left(s+\Delta_{i j}\right)$ is an optimal solution for the state $s+\Delta_{i j}$, for $\exists k, l$,

$$
\begin{aligned}
& J^{1}\left(s+\Delta_{m n}, u\left(s+\Delta_{i j}\right)\right) \leq J^{1}\left(s+\Delta_{m n}, u\left(s+\Delta_{i j}\right)+\Delta_{k l}\right) \\
& \Leftrightarrow\left(1-\alpha_{k l}\right) c_{w}(k, l)+\alpha_{k l} c_{r} \leq \int_{B}^{\infty} c_{o}(\tau)(\tau-B) \mathrm{d} F^{(u+1) *}(\tau) \\
& -\int_{B}^{\infty} c_{o}(\tau)(\tau-B) \mathrm{d} F^{u *}(\tau)+\gamma \mathbb{E}\left\{v \left(s+\Delta_{m n}-\Delta_{k l}\right.\right. \\
& \left.\left.-u\left(s+\Delta_{i j}\right)-z_{1}+d\right)-v\left(s+\Delta_{m n}-u\left(s+\Delta_{i j}\right)-z_{2}+d\right)\right\},
\end{aligned}
$$

where $z_{1} \sim B\left(s-u\left(s+\Delta_{i j}\right), \alpha\right)$ and $z_{2} \sim B\left(s-u\left(s+\Delta_{i j}\right)+\Delta_{i j}, \alpha\right)$.

If we assume $u_{k l}^{*}<u_{k l}^{\prime}$ for $\exists k, l$, where $u_{k l}^{\prime} \in u\left(s+\Delta_{m n}\right)$ and $u_{k l}^{*} \in u\left(s+\Delta_{i j}\right)$, then

$$
\begin{aligned}
& J^{2}\left(s+\Delta_{m n}, u\left(s+\Delta_{i j}\right)\right)>J^{2}\left(s+\Delta_{m n}, u\left(s+\Delta_{i j}\right)+\Delta_{k l}\right) \\
& \Leftrightarrow\left(1-\alpha_{k l}\right) c_{w}(k, l)+\alpha_{k l} c_{r}>\int_{B}^{\infty} c_{o}(\tau)(\tau-B) \mathrm{d} F^{(u+1) *}(\tau) \\
& -\int_{B}^{\infty} c_{o}(\tau)(\tau-B) \mathrm{d} F^{u *}(\tau) \\
& +\gamma \mathbb{E}\left\{v\left(s+\Delta_{m n}-\Delta_{k l}-u\left(s+\Delta_{i j}\right)-z_{3}+d\right)\right. \\
& \left.-v\left(s+\Delta_{m n}-u\left(s+\Delta_{i j}\right)-z_{4}+d\right)\right\},
\end{aligned}
$$

where $z_{3} \sim B\left(s+\Delta_{m n}-\Delta_{k l}-u\left(s+\Delta_{i j}\right), \alpha\right)$ and $z_{4} \sim B\left(s+\Delta_{m n}-u\left(s+\Delta_{i j}\right), \alpha\right)$. From (5) and (6),

$$
\begin{gathered}
v\left(s+\Delta_{m n}-\Delta_{k l}-u\left(s+\Delta_{i j}\right)-z_{1}+d\right)-v\left(s+\Delta_{m n}-u\left(s+\Delta_{i j}\right)-z_{2}+d\right)> \\
v\left(s+\Delta_{m n}-\Delta_{k l}-u\left(s+\Delta_{i j}\right)-z_{3}+d\right)-v\left(s+\Delta_{m n}-u\left(s+\Delta_{i j}\right)-z_{4}+d\right) .
\end{gathered}
$$

However, because of the monotonicity of the value function shown in Lemma 1, Inequality (7) does not hold.

Therefore, the assumption $u_{k l}^{*}<u_{k l}^{\prime}$ does not hold, and $u_{k l}^{*} \geq u_{k l}^{\prime}$ for all $k, l$, where $u_{k l}^{\prime} \in u\left(s+\Delta_{m n}\right)$ and $u_{k l}^{*} \in u\left(s+\Delta_{i j}\right)$ if $\left(1-\alpha_{i j}\right) c_{w}(i, j)+c_{r} \alpha_{i j} \geq$ $\left(1-\alpha_{m n}\right) c_{w}(m, n)+c_{r} \alpha_{m n}$. 


\section{REFERENCES}

[1] J.M. Alden and R.L. Smith, Rolling horizon procedures in nonhomogeneous Markov Decision Processes. Oper. Res. 40 (1992) 183-194.

[2] D.P. Bertsekas, Dynamic programming and optimal control, Vol. 2, 2nd edition. Athena Scientific, Belmont, MA (2007).

[3] D.P. Bertsekas and D.A. Castanon, Rollout algorithms for stochastic scheduling problems. J. Heuristics 5 (1999) 89-108.

[4] T. Cheevaprawatdomrong and R.L. Smith, Infinite horizon production scheduling in timevarying systems under stochastic demand. Oper. Res. 52 (2004) 105-115.

[5] H.S. Chang, M.C. Fu, J. Hu and S.I. Marcus, An adaptive sampling algorithm for solving Markov Decision Processes. Oper. Res. 53 (2005) 126-139.

[6] F. Dexter, A. Macario and R.D. Traub, Which algorithm for scheduling add-on elective cases maximizes operating room utilization? Use of bin packing algorithms and fuzzy constraints in operating room management. Anesthesia and Analgesia 90 (1999) 980-988.

[7] J. Everett, A decision support simulation model for the management of an elective surgery waiting system. Health Care Manag. Sci. 5 (2002) 89-95.

[8] Y. Gerchak, D. Gupta and M. Henig, Reservation planning for elective surgery under uncertain demand for emergency surgery. Manag. Sci. 42 (1996) 321-334.

[9] L. Green, S. Savin and B. Wang, Managing patient service in a diagnostic medical facility. Oper. Res. 54 (2006) 11-25.

[10] D. Gupta, Surgical Suites Operations Management. Prod. Oper. Manag. 16 (2007) 689-700.

[11] E. Hans, G. Wullink, M.V. Houdenhoven and G. Kazemier, Robust surgery loading. Eur. J. Oper. Res. 185 (2008) 1038-1050.

[12] O. Hernandez-Lerma and J.B. Lasserre, Error bounds for rolling horizon policies in discretetime Markov control processes. IEEE Trans. Autom. Control 35 (1990) 1118-1124.

[13] J. Hurst and L. Sicilliani, Tackling excessive waiting times for elective surgery: A comparison of policies in twelve OECD countries. OECD Health Work. Pap. 2003 (2003).

[14] M. Kearns, Y. Mansour and A.Y. Ng, A sparse sampling algorithm for near-optimal planning in large Markov Decision Processes. Machine Learn. 49 (2002) 193-208.

[15] M. Lans, E. Hans, J.L. Hurink, G. Wullink, M. Houdenhoven and G. Kazemier, Anticipating urgent surgery in operating room departments, Working Paper, University of Twente, The Netherlands (2006).

[16] L. Liu and X. Liu, Dynamic and static job allocation for multi-server systems. IIE Trans. 30 (1998) 845-854.

[17] N. Liu, S. Ziya and V.G. Kulkarni, Dynamic scheduling of outpatient appointments under patient no-shows and cancellations. Manufacturing and Service Oper. Manag. Published online (2009).

[18] W.S. Lovejoy and Y. Li, Hospital operating room capacity expansion. Manag. Sci. 48 (2002) 1369-1387.

[19] A.D. MacCormick, W.G. Collecutt and B.R. Parry, Prioritizing patients for elective surgery: A systematic review. ANZ J. Surgery, 73 (2003) 633-642.

[20] E. Marcon, S. Kharraja and G. Simonnet, The operating theatre planning by the follow-up of the risk of no realization. Int. J. Prod. Econ. 85 (2003) 83-90.

[21] D. Min and Y. Yih, An elective surgery scheduling problem considering patient priority. Comput. Oper. Res. 37 (2010) 1091-1099.

[22] P.M. Mullen, Prioritizing waiting lists: how and why? Eur. J. Oper. Res. 150 (2003) 32-45.

[23] K. Muthuraman and M. Lawley, A stochastic overbooking model for outpatient clinical scheduling with no-shows. IIE Trans. 40 (2008) 820-837.

[24] M. Olivares, C. Terwiesch and L. Cassorla, Structural estimation of the newsvendor model: an application to reserving operating room time. Manag. Sci. 54 (2008) 41-55.

[25] J. Patrick, M. Puterman and M. Queyranne, Dynamic multi-priority patient scheduling for a diagnostic resource. Oper. Res. 56 (2008) 1507-1525. 
[26] W.B. Powell, Approximate dynamic programming: Solving the curses of dimensionality. John Wiley and Sons, Inc., New York (2007).

[27] I.E. Schochetman and R.L. Smith, Infinite horizon optimization. Math. Oper. Res. 14 (1989) $559-574$.

[28] L.R. Smith and Q.R. Zhang, Infinite horizon production planning in time-varying systems with convex production and inventory costs. Manag. Sci. 44 (1998) 1313-1320.

[29] B. Sobolev, L. Kuramoto, A. Levy and R. Hayden, Cumulative incidence for wait-list death in relation to length of queue for coronary-artery bypass grafting: a cohort study. J. Cardiothoracic Surgery 1 (2006) 1-10.

[30] B. Sobolev and L. Kuramoto, Analysis of waiting-time data in health services research. New York, Springer (2007).

[31] B. Sobolev, V. Sanchez, L. Kuramoto, A.R. Levy M. Schechter and M. FitzGerald, Evaluation of booking systems for elective surgery using simulation experiments. Healthcare Policy 3 (2008) 113-124.

[32] D.P. Strum, L.G. Varga, J.H. May and G. Bashein, Surgical suite utilization and capacity planning: a minimal cost analysis model. J. Medical Systems 21 (1997) 309-322.

[33] A. Testi, E. Tanfani and G. Torre, A three-phase approach for operating theatre schedules. Health Care Manag. Sci. 10 (2007) 163-172.

[34] S. Thompson, M. Nunez, R. Garfinkel and M.D. Dean, Efficient short-term allocation and reallocation of patients to floors of a hospital during demand surges. Oper. Res. 57 (2009) 261-273.

[35] C. Vasilakis, B. Sobolev, L. Kuramoto and A. Levy, A simulation study of scheduling clinic appointments in surgical care: individual surgeon versus pooled lists. J. Oper. Res. Soc. 58 (2007) 202-211. 